\title{
Pulseless Electrical Activity: Detection of Underlying Causes in a Prehospital Setting
}

\author{
Senne Van den Bempt ${ }^{a} \quad$ Lina Wauters ${ }^{a} \quad$ Philippe Dewolf ${ }^{a}$ b \\ ${ }^{a}$ Department of Emergency Medicine, University Hospitals of Leuven, Leuven, Belgium; ${ }^{b}$ Department of Public \\ Health and Primary Care, KU Leuven - University, Leuven, Belgium
}

\section{Significance of the Study}

- Clinical and anamnestic signs remain vital and should be linked to 4H's and 4T's.

- Ultrasonography should be used to detect underlying causes of cardiac arrest.

- Cerebral oximetry could become an important tool in prognostication.

- Detection of cardiac movement by ultrasonography has important prognostic implications.

\section{Keywords}

Pulseless electrical activity · Out-of-hospital cardiac arrests · Clinical decision-making

\section{Abstract}

The proportion of out-of-hospital cardiac arrests (OHCAs) with pulseless electrical activity (PEA) as initial rhythm is increasing. PEA should be managed by identifying the underlying cause of the arrest and treating it accordingly. This often poses a challenge in the chaotic prehospital environment with only limited resources available. The aim of this study was to review the diagnostic tools available in a prehospital setting, and their interpretation during cardiac arrest (CA) with PEA as initial rhythm. A systematic literature search of the PubMed database was performed. Articles were assessed for eligibility by title, abstract, and full text. Ultrasonography has become a great asset in detecting underlying causes, and a variety of protocols have been pro- posed. There are currently no studies comparing these protocols regarding their feasibility and their effect on patient survival. Further research concerning the relationship between electrocardiogram characteristics and underlying causes is required. Limited evidence suggests a role for point-of-care testing in detecting hyperkalemia and a role for capnography in the diagnosis of asphyxia CA. Multiple studies describe a prognostic potential. Although evidence about the prognostic potential of cerebral oximetry in OHCA is accumulating, its diagnostic potential is still unknown. In the management of $\mathrm{OHCA}$, anamnestic and clinical information remains the initial source of information in search for an underlying cause. Ultrasonographic evaluation should be performed subsequently, both for detecting an underlying cause and discriminating between true PEA and pseudo PEA. Comparative studies are required to identify the best ultrasonographic protocol, which can be included in resuscitation guidelines.

(C) 2020 The Author(s)

Published by S. Karger AG, Basel karger@karger.com www.karger.com/mpp

Karger $\stackrel{\text { ' }}{5}$

BOPEN ACCESS
(C) 2020 The Author(s)

Published by S. Karger AG, Basel

This is an Open Access article licensed under the Creative Commons Attribution-NonCommercial-4.0 International License (CC BY-NC) (http://www.karger.com/Services/OpenAccessLicense), applicable to the online version of the article only. Usage and distribution for commercial purposes requires written permission.
Philippe Dewolf

Department of Emergency Medicine

University Hospitals of Leuven

Herestraat 49, BE-3000 Leuven (Belgium)

philippe.dewolf@uzleuven.be 


\section{Introduction}

Sudden cardiac arrest (CA) is a leading cause of death in the Western world. In Europe, annual incidences up to 119 per 100,000 adults have been reported [1]. Despite considerable efforts to improve the various links in the chain of survival, out-of-hospital cardiac arrest (OHCA) remains associated with an exceptionally poor prognosis [2-6]. As the proportion of CAs with pulseless electrical activity (PEA) as initial rhythm is increasing, this particular subtype is the main subject of this review [4].

PEA is defined as the absence of a palpable pulse, even though the electrocardiogram (ECG) demonstrates a synchronized electrical rhythm $[7,8]$. In true PEA, no cardiac movement can be detected. The heart is at a complete standstill despite a normal electrical rhythm. This should however be differentiated from pseudo PEA in which some cardiac movement is still present although not powerful enough to produce adequate circulation. This is often caused by a severe shock state (such as hypovolemia or obstruction of cardiac output) [9]. If the underlying cause is not reversed, the heart continues to pump despite an ongoing depletion of oxygen and metabolic supplies. Eventually, with ongoing depletion and deterioration, myocardial contractions disappear completely, resulting in true PEA $[7,9]$. True PEA can thus be regarded as the final common pathway of pseudo PEA.

Even though prognosis has increased over the years, PEA still has an estimated 1-month survival of merely $4.9-6.8 \%[4,10,11]$. In contrast to arrhythmogenic CAs, where the main treatment strategy consists of shock delivery, PEA is managed by diagnosis and treatment of the underlying cause $[12,13]$. The most frequent reversible causes of PEA are hypoxia, hypovolemia, hypo-/hyperkalemia, hypo-/hyperthermia, cardiac tamponade, tension pneumothorax, thrombosis (both coronary and pulmonary), and toxins [12].

Early identification of these reversible etiologies during resuscitation is key, as it allows health care professionals to optimize treatment, and thus increase chances of survival. However, the limited availability of diagnostic equipment in prehospital care poses a challenge in the identification of the cause of the arrest, as emergency physicians can often only rely on the patient's medical history and clinical examination.

Over the last few years, technology has improved and an increasing number of diagnostic tools have become available for use in a prehospital CA setting. Not only are these tools helpful in detecting an underlying cause, they can also assist emergency physicians in clinical decision-

PEA: Detection of Underlying Causes in a Prehospital Setting making by determining the expected prognosis $[2,14-$ 24]. Several studies have been published investigating the usefulness of QRS characteristics on ECG to discriminate between groups of causal mechanisms $[25,26]$. Another tool that has been used extensively to monitor OHCAs is capnography. A magnitude of studies have been conducted on its usefulness and its ability to predict return of spontaneous circulation (ROSC) [14, 27]. Ultrasonography has the potential to become an important asset in CA settings as well, even more so since the development of wireless, handheld devices. Finally, portable cerebral oxygenation monitoring devices have become a hot topic in research on OHCA. This study reviews the current literature regarding the available diagnostic tools in a prehospital setting and their application and interpretation during a CA with pulseless electric activity as initial rhythm.

\section{Search Strategy}

A systematic literature search of the PubMed database was performed (Fig. 1). The database was searched from inception until October 2019. All titles and abstracts were screened for their relevance. After this initial screening, full-text articles were assessed for eligibility. Case reports and non-English articles were not included. The search strategy combined Medical Search Headings and Subheadings (MeSH) terms (in PubMed), with Boolean operators "AND" and "OR" to capture all relevant article suggestions. Table 1 shows the search terms for each topic. Abstracts without full-text articles were excluded. The references of the identified articles were screened for additional eligible articles. The search strategy resulted in 926 hits in total. After initial screening, 44 full-text articles were assessed for eligibility, of which 37 articles were included in this review. Of these 37 articles, 3 were found concerning ECG. Seven articles were found on the subject of capnography. A total of 17 articles were used to review ultrasonography. Six articles concerning cerebral oximetry were included. Another 6 articles were identified describing point-of-care testing (POCT).

\section{H's and 4T's}

The current approach to PEA consists of unveiling and treating the underlying cause using anamnestic information and clinical signs. The most frequent reversible causes of CA with PEA as the initial rhythm are commonly referred to as the 4H's and 4T's: hypoxia, hypovo- 
Searched "(("Electrocardiography" [MeSH]) AND "("Heart Arrest/etiology" [MeSH] OR "Out-ofHospital Cardiac Arrest/etiology" [MeSH])) AND

"Cardiopulmonary resuscitation" [MeSH]"

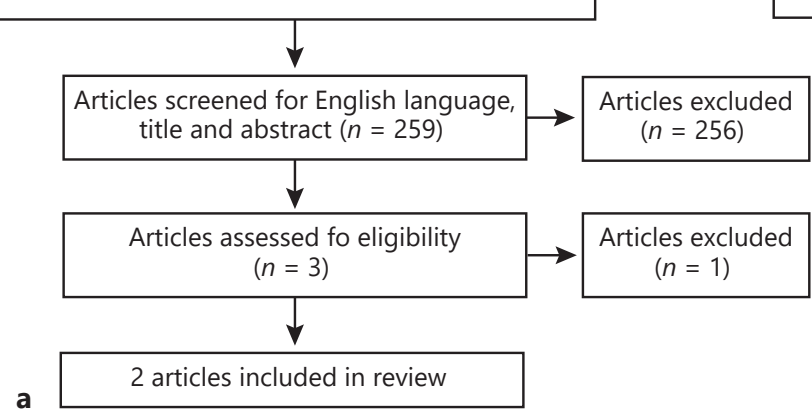

Searched "(((("Prognosis" [MeSH]) OR "Outcome Assessmen (Health Care)" [MeSH]) AND "Capnography" [MeSH])

AND (("Heart Arrest" [MeSH]) OR "Out-of-Hospital

Cardiac Arrest" [MeSH]) AND "Cardiopulmonary Resuscitation" [Mesh]"

Searched "((("Heart Arrest/etiology" [MeSH]) AND

"Cardiopulmonary Resuscitation" [MeSH]) AND

("Echocardiography" [MeSH] OR "Ultrasonography" [Mesh])

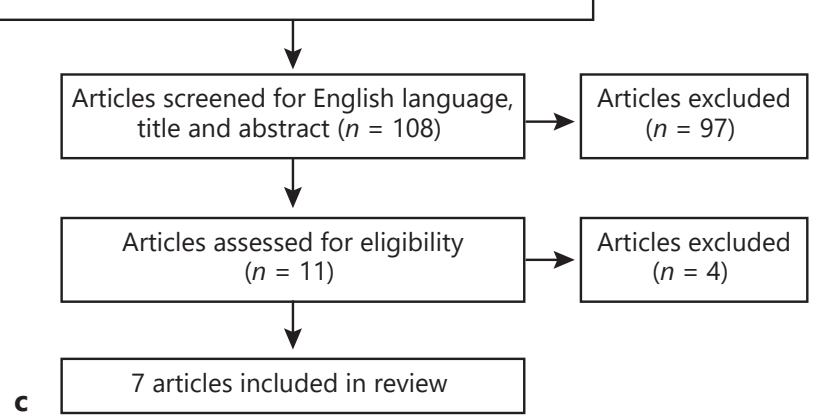

Searched "Focused echocardiographic evaluation in life support"

b
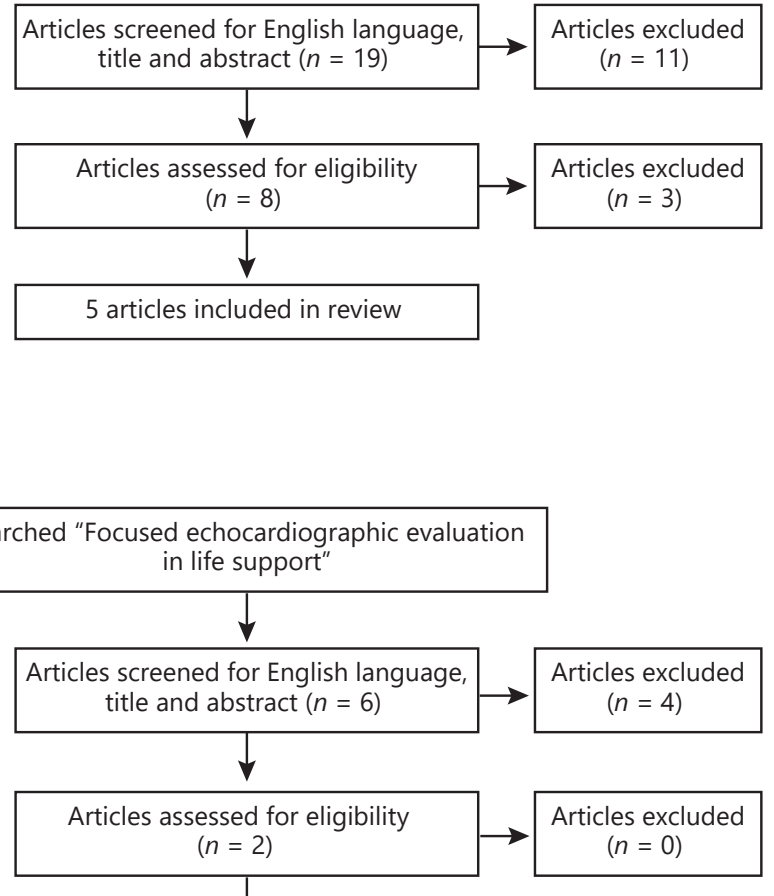

d

2 articles included in review

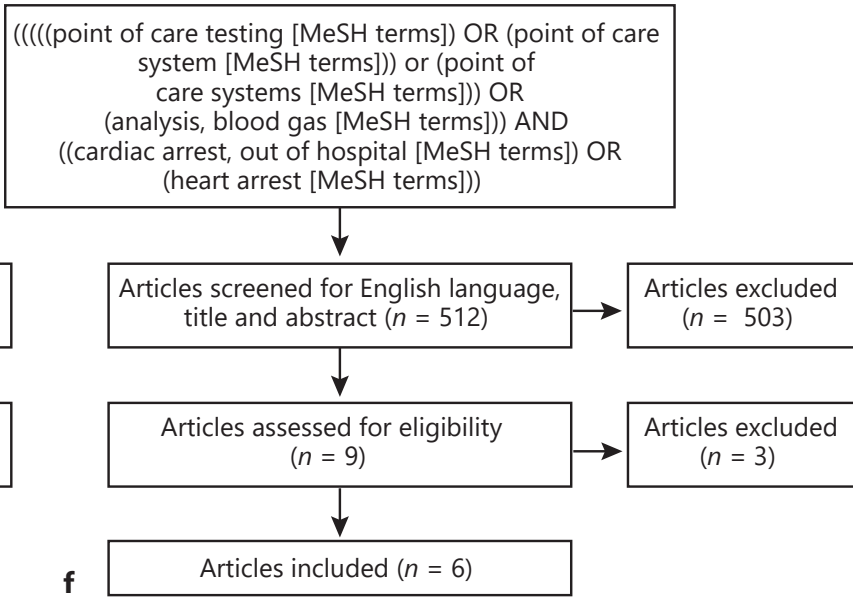

Searched "("Oximetry" [MeSH]) AND "Out-ofHospital Cardiac Arrest" [MeSH]"

e

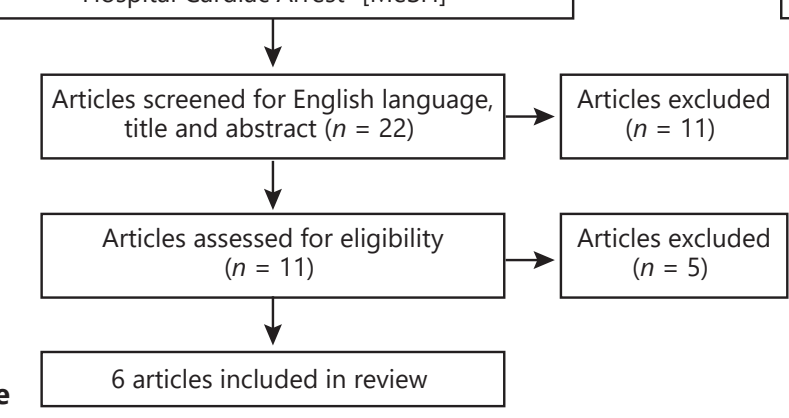

Fig. 1. Search methodology. a Search methodology for ECG. b Search methodology for capnography. c Search methodology for ultrasonography. d Search methodology for FEEL. e Search methodology for cerebral oximetry. f Search methodology for POCT. FEEL, Focused Echocardiographic Evaluation in Life support; POCT, pointof-care testing. 
Table 1. Search terms

\begin{tabular}{|c|c|}
\hline Subject & Search term \\
\hline ECG & $\begin{array}{l}\text { ((“Electrocardiography”[MeSH] ) AND (“Heart Arrest/etiology”[MeSH] OR “Out-of-Hospital Cardiac Arrest/ } \\
\text { etiology”[MeSH])) AND “Cardiopulmonary Resuscitation”[MeSH] }\end{array}$ \\
\hline Capnography & $\begin{array}{l}\text { (((“Prognosis”[MeSH] ) OR “Outcome Assessment (Health Care)”[MeSH] }) \text { AND “Capnography”[MeSH] }) \text { AND } \\
\text { (("Heart Arrest”[MeSH] }) \text { OR “Out-of-Hospital Cardiac Arrest”[MeSH] }) \text { AND “Cardiopulmonary } \\
\text { Resuscitation”[MeSH] }\end{array}$ \\
\hline FEEL & Focused echocardiographic evaluation in life support \\
\hline Cerebral oximetry & (“Oximetry”[MeSH]) AND “Out-of-Hospital Cardiac Arrest”[MeSH] \\
\hline POCT & $\begin{array}{l}((((\text { Point of care testing }[\mathrm{MeSH} \text { terms] }) \text { OR (point of care system[MeSH terms] })) \text { OR (point of care } \\
\text { systems[MeSH terms] }) \text { OR (analysis, blood gas[MeSH terms] }) \text { AND ((cardiac arrest, out of hospital[MeSH } \\
\text { terms]) OR (heart arrest[MeSH terms] }))\end{array}$ \\
\hline
\end{tabular}

ECG, electrocardiogram; MeSH, Medical Search Headings and Subheadings; FEEL, Focused Echocardiographic Evaluation in Life support; POCT, point-of-care testing.

lemia, hypo/hyperkalemia, hypo/hyperthermia, cardiac tamponade, tension pneumothorax, thrombosis (both coronary and pulmonary), and toxins [12]. However, in the case of potassium disorders, it appears that especially hyperkalemia results in PEA [28].

Treatment of the 4H's and 4T's is described extensively in the 2015 ERC Guidelines [12]. Hypoxia is treated by providing adequate oxygen delivery to the lungs. Hypovolemia requires adequate hemostasis and administration of IV fluids. Hyperkalemia should be managed by administering calcium, glucose/insulin, and sodium bicarbonate. In the case of hypothermia CA, patients should be rewarmed, while in contrast, hyperthermic patients should be cooled. Resuscitative thoracotomy or pericardiocentesis can be lifesaving in the case of cardiac tamponade. In the presence of a tension pneumothorax, needle decompression can be performed but should be followed by thoracotomy. In patients suffering from CA caused by pulmonary embolism, fibrinolytic therapy can be administered. Finally, the treatment of toxic CA depends on the causal toxic agent [12].

\section{Instruments}

\section{Electrocardiogram}

ECG is an important instrument in CA settings. It can be used to differentiate between initial rhythms. Furthermore, it has been suggested that ECG could help to diag- nose the underlying cause of PEA. Littmann et al. [25] stated on a theoretical basis that a small-complex PEA on ECG signifies a mechanical cause while a wide-complex PEA indicates a metabolic cause. However, a study by Bergum et al. [26] could not demonstrate a correlation between ECG patterns and the causal mechanism of PEA. They suggested that the aberrant depolarization resulting from metabolic deterioration of myocardial fibers during CA can cause widened ECG patterns.

Even though Bergum et al. [26] stated that ECG cannot help to determine the causal mechanism of PEA, it appears to be able to predict ROSC. Skjeflo et al. [29] discovered that 3-6 min prior to ROSC the heart rate on ECG during CPR starts to increase while QRS width appears to decrease. The heart rate of patients who did not achieve ROSC remained constant or decreased, and QRS width increased or remained unchanged [29].

\section{Capnography}

In end-tidal capnography, expiratory $\mathrm{CO}_{2}$ levels reflect tissue metabolism, tissue and lung perfusion, and alveolar ventilation [27]. Detection of $\mathrm{CO}_{2}$ can thus be used to verify the correct placement of the endotracheal tube and to monitor the effectiveness of chest compressions [8]. An end-tidal $\mathrm{CO}_{2}\left(\mathrm{ETCO}_{2}\right)$ value below $10-15 \mathrm{~mm}$ $\mathrm{Hg}$ indicates an insufficient quality of chest compressions [14]. A sudden $\mathrm{ETCO}_{2}$ increase to $>40 \mathrm{~mm} \mathrm{Hg}$ during CPR is indicative for ROSC [14]. Furthermore, Pokorná et al. [27] discovered that a sudden $\mathrm{ETCO}_{2}$ increase of at 
least $10 \mathrm{~mm} \mathrm{Hg}$ during advanced life support (ALS) can also indicate ROSC.

Not only can capnography be used to detect ROSC, it can also predict the likeliness of ROSC. Poon et al. [14] found that an $\mathrm{ETCO}_{2}$ level $>10 \mathrm{~mm} \mathrm{Hg} 3 \mathrm{~min}$ after intubation can predict ROSC with odds ratio 18.16. In a study by Grmec et al. [17], all patients suffering from a CA with shockable rhythm that achieved ROSC had an initial $\mathrm{ETCO}_{2}>10 \mathrm{~mm} \mathrm{Hg}$ as well. Pearce et al. [18] confirmed that higher initial levels of $\mathrm{ETCO}_{2}$ were indeed associated with ROSC but not with survival to discharge. Although $\mathrm{ETCO}_{2}$ levels appeared to be higher in survivors to discharge compared to non-survivors, the difference was not statistically significant [18]. The same trend was observed by Salen et al. [19] where median $\mathrm{ETCO}_{2}$ levels were higher in the ROSC group ( $35 \mathrm{~mm} \mathrm{Hg}$ ) compared to the nonsurvivor group (13.7 $\mathrm{mm} \mathrm{Hg}$ ). There were no survivors with $\mathrm{ETCO}_{2}$ levels below $16 \mathrm{~mm} \mathrm{Hg}$ [19].

Grmec et al. [17] also found that CA patients who suffered from asphyxia (initial rhythm PEA or asystole) had higher $\mathrm{ETCO}_{2}$ levels than those with an initial shockable rhythm. Mean $\mathrm{ETCO}_{2}$ values were $66.4 \pm 17$ and $16.5 \pm$ $9.2 \mathrm{~mm} \mathrm{Hg}$ for asphyxial and primary $\mathrm{CA}$, respectively. This was explained by accumulation of $\mathrm{CO}_{2}$ in the lungs. Cessation of breathing results in cessation of alveolar washout of $\mathrm{CO}_{2}$. However, circulatory $\mathrm{CO}_{2}$ delivery to the lungs is initially preserved, which causes the $\mathrm{CO}_{2}$ to accumulate and results in initial high $\mathrm{ETCO}_{2}$ values [17, 30, 31]. This also explains why no significant difference in initial $\mathrm{ETCO}_{2}$ could be demonstrated between patients achieving and not achieving ROSC in asphyxia CA [17].

\section{Ultrasonography}

Evidence is accumulating regarding the benefits of ultrasonography use during CPR. First, detection of cardiac contractions with ultrasonography makes differentiation between true and pseudo PEA possible $[2,8]$. This has important implications as pseudo PEA has a much better prognosis than true PEA $[2,9,20]$. The rate of survival to hospital admission in PEA with cardiac movement is $55 \%$, while in PEA without cardiac movement it is only $8 \%[2,20]$. Pseudo PEA should also potentially be managed differently as it was shown that administration of 20 IU vasopressin during a prolonged cessation of chest compressions of $15 \mathrm{~s}$ could significantly improve its outcome [32]. Since this treatment strategy was however investigated in a relatively small study population, further research is required to confirm these findings.

Second, detection of spontaneous cardiac movement by ultrasonography could be predictive of ROSC (note: reference identified by manual PubMed search using a different search term) [33]. Finally, some of the underlying causes of PEA that are difficult to detect clinically can easily be detected by ultrasonography [34]. Echocardiographic signs of cardiac tamponade include presence of pericardial effusion, right atrial or ventricular diastolic collapse, and non-collapsible inferior vena cava. These signs can easily be detected using a subxiphoidal or parasternal view $[35,36]$. Hypovolemia can also be diagnosed by detecting an underfilled right or left ventricle or a collapsed inferior vena cava [35-37]. Sonographical signs of tension pneumothorax include absence of lung sliding when positioning a probe at the midclavicular line at the second intercostal space. The absence of lung sliding is a sign of high sensitivity (92.3\%) and specificity (99.6\%) and allows a diagnosis to be made within $30 \mathrm{~s}$ [35]. However, parietal emphysema can hide a pneumothorax by mimicking pleural motion. This is why bilateral examination and comparison should be performed [13].

Emboli can rarely be visualized directly [38]. Pulmonary embolism should be suspected when echocardiography shows a dilated right ventricle and a flattened left ventricle or when normal wall function at the apex combined with right ventricle dysfunction at the mid-free wall is seen $[13,35,39,40]$. The latter is known as the McConnell sign. These sonographic signs have low sensitivity (and thus have limited value as a screening tool), but high specificity, making it very useful in therapeutic decisionmaking [35, 40-44].

Several studies have concluded that ultrasonography during resuscitation is indeed feasible and does not interfere with the management of ALS [38, 45, 46]. A variety of sonographic protocols have been suggested for use during CPR. The most notable sonographic protocols are mentioned in Table 2.

One widely used protocol is Focused Echocardiographic Evaluation in Life support (FEEL) which was developed to detect underlying etiologies during CPR. In this protocol, a subxiphoidal probe position is used to obtain imaging of the heart within a $10 \mathrm{~s}$ time frame, during a pulse check between two cycles of ALS [34]. This probe position is preferred as it leads to better imaging quality than other views [20] and should be brought in position while chest compressions are being delivered. A full 4-chamber view should be obtained and analyzed while chest compressions resume. If the subxiphoidal position fails to adequately visualize the heart, other probe positions should be used; the parasternal window, long-axis, short-axis, or finally the apical 4 -chamber view. The FEEL protocol allows diagnosis of hypovolemia, cardiac tam- 
Table 2. Summary of ultrasonographic protocols

\begin{tabular}{|c|c|c|c|c|}
\hline $\begin{array}{l}\text { Probe } \\
\text { positioning }\end{array}$ & Subxiphoidal & $\begin{array}{l}\text { Subxiphoidal (parasternal, apical) } \\
\text { Second intercostal space on } \\
\text { midclavicular line }\end{array}$ & $\begin{array}{l}\text { Epigastric (apical) } \\
\text { Parasternal at second or third intercostal space } \\
\text { Left parasternal at third and fourth intercostal space } \\
\text { Abdomen } \\
\text { Lower limbs }\end{array}$ & $\begin{array}{l}\text { Subxiphoidal (parasternal) } \\
\text { Anterior and lateral chest } \\
\text { Abdomen } \\
\text { Proximal lower limbs } \\
\text { Anterior neck }\end{array}$ \\
\hline Time frame & Pulse check (max. $10 \mathrm{~s}$ ) & $\begin{array}{l}\text { Cardiac examination: pulse check } \\
\text { (max. } 10 \mathrm{~s} \text { ) } \\
\text { Lung examination: } 30 \mathrm{~s}\end{array}$ & Unspecified & $\begin{array}{l}\text { Cardiac examination: pulse } \\
\text { check (max. } 10 \mathrm{~s} \text { ) }\end{array}$ \\
\hline
\end{tabular}

FEEL, Focused Echocardiographic Evaluation in Life support; CAUSE, Cardiac Arrest Ultra Sound Examination; PEA, pulseless electrical activity.

ponade, and pulmonary embolism [34]. Although additional research on its effect on patient outcome is recommended, the feasibility of the FEEL protocol was demonstrated in a prehospital setting. Implementation of sonographic evaluation using FEEL resulted in a change in therapy in $89 \%$ of patients undergoing CPR [20].

The Cardiac Arrest UltraSound Examination (CAUSE) protocol aims to diagnose the most common and easily reversible underlying causes of non-arrhythmogenic CA: hypovolemia, cardiac tamponade, pulmonary embolism, and tension pneumothorax. In this protocol, two sonographic perspectives of the thorax are used: a cardiac 4-chamber view and a view of the lung and pleura. The latter view is obtained by placing the probe at the second intercostal space on the midclavicular line. The cardiac and pericardial 4 chamber view can be obtained using a subcostal, parasternal, or apical probe position. The CAUSE protocol states that a cardiac view should be obtained first, moving on to the lung and pleural view if a cardiac cause could not be detected. This is because the cardiac view takes the least amount of time to complete, does not interfere with chest compressions and can detect three possible causes at once [35].

Testa et al. [36] proposed the P.E.A. protocol yet another sonographic protocol for PEA which is an acronym for the proposed probe positions, parasternal, epigastric, and abdominal. With the parasternal probe position, pneumothorax, pleural effusion, and wet or dry lungs can be detected. The epigastric position can demonstrate pericardial effusion, inferior vena cava filling and filling status of the left and right ventricle, and their wall motion. The abdominal probe position is used to detect the presence of an abdominal aneurysm or dissection, peritoneal effusion, bowel occlusion or perforation, and deep venous thrombosis. Cardiac evaluation should be performed first. The second probe position depends on the findings of the cardiac examination. If hypovolemia is suspected on cardiac evaluation (collapsed IVC), an abdominal scan should be performed next. If cardiac tamponade is found, parasternal views should be obtained to detect aortic dissection. If an enlarged, hypokinetic right ventricle is detected, pulmonary embolism is suspected and a compression sonography of the lower limbs should be carried out. If a hypokinetic, distended left ventricle is detected, the lungs should be checked for pleural effusion or signs of a wet lung [36]. Testa et al. [36] state that the P.E.A. protocol can easily be applied during ALS and cite other studies $[34,46]$ to demonstrate that ultrasonography does not delay CPR.

In 2017, The International Federation for Emergency Medicine (IFEM) Ultrasound Special Interest Group (USIG) published another sonographic protocol for hypotension and $\mathrm{CA}$, the $\mathrm{SHoC}$ protocol. This protocol consists of core, supplementary, and additional views. The sonographic views in the "core" category should be performed routinely in all patients. In a CA setting, the core category consists of a subxiphoid view or a parasternal cardiac view if the former could not obtain adequate images. These views can both be performed during the pauses used for rhythm checks and thus cause only mini- 
mal interruption of chest compressions. Supplementary views should be obtained in all patients where this could lead to additional information without delaying CPR. These consist of inferior vena cava and lung views. Additional views should only be performed when clinically indicated. The SHoC protocol recommends the 4-F's approach for interpreting the obtained sonographic images, fluid, form, function, and filling. For cardiac views, this means checking for pericardial effusion (fluid), interpreting the cardiac dimensions (form), and judging the contractility and valve functioning (function). For inferior vena cava, this means assessing whether the vessel is dilated and non-collapsing or small and collapsing, while the lungs should be checked for B-lines and pleural effusion, indicating congestive heart failure (filling). The lung views can also detect pneumothorax by noticing an absence of the lung sliding sign. Additional views can, for example, consist of checking the lower limbs for DVT when pulmonary embolus is suspected, or checking the abdomen for rupture of an aortic aneurysm or another cause of blood loss when hypovolemia is detected [37].

\section{Cerebral Oximetry}

Evidence is accumulating for monitoring the patient's status during CPR by cerebral oximetry using near infrared spectroscopy. A monitor with a light source is placed on the forehead of the patient undergoing CPR. This takes on average $15 \mathrm{~s}$, without interrupting CPR. The light emitted by the light source travels through the skin, skull, and brain tissue of the patient. The device measures the difference in light absorption between oxygenated and non-oxygenated hemoglobin and calculates the tissue oxygenation based on the amount of light arriving at the detectors. In brain tissue, normal oxygenation values range from 60 to $80 \%$ [47].

Prosen et al. [22] investigated the cerebral oxygen saturation $\left(\mathrm{rSO}_{2}\right)$ level and its change during CPR for ROSC and no-ROSC patients. The initial $\mathrm{rSO}_{2}$ levels on arrival of the emergency medical services were undetectable $(<15 \%)$ in $52 \%$ of patients achieving ROSC and $59 \%$ of patients not achieving ROSC. During CPR, $\mathrm{rSO}_{2}$ levels rose in all cases. However, a significantly higher rise is seen in patients achieving ROSC (median rise of $22 \%$ in ROSC vs. $14 \%$ in no-ROSC). Furthermore, minutes before ROSC a significant and rapid rise in $\mathrm{rSO}_{2}$ levels is seen. In this study, the mean $\mathrm{rSO}_{2}$ value at ROSC was $47 \%$, while the mean peak $\mathrm{rSO}_{2}$ level in no-ROSC patients was only $31 \%$, indicating that ROSC might be predicted by a significant rise in $\mathrm{rSO}_{2}$ prior to occurrence. On the contrary, persistently low $\mathrm{rSO}_{2}$ levels during CPR pre- 
dicted poor neurological outcomes [22]. A systematic review and meta-analysis by Sanfilippo et al. [23] also concluded that both higher initial rSO2 levels and higher average $\mathrm{rSO}_{2}$ levels are associated with greater chances of achieving ROSC after CA. Singer et al. [24] also observed a correlation between higher mean $\mathrm{rSO}_{2}$ levels and ROSC after CA. Their study also showed that ROSC was rarely achieved when $\mathrm{rSO}_{2}$ remained below 30\% [24]. A study by Fukuda et al. [15] found that an initial $\mathrm{rSO}_{2}$ level of $26 \%$ or less could predict no-ROSC with a sensitivity of $89 \%$, a specificity of $56 \%$, a positive predictive value of $87 \%$, and a negative predictive value of $60 \%$.

Not only do $\mathrm{rSO}_{2}$ levels predict ROSC, they can also predict neurological outcome. Nishiyama et al. [48] investigated the relationship between $\mathrm{rSO}_{2}$ levels at hospital arrival after OHCA and neurological outcome after 90 days. They found that with increasing lower $\mathrm{rSO}_{2}$ values, the percentage of patients with good 90-day neurological outcome increased proportionally, irrespective of the ROSC status upon arrival at the hospital [48]. In a previous study, they found an optimal cutoff $\mathrm{rSO}_{2}$ value for good neurological outcome of $>42 \%$ [49].

\section{Point-of-Care Testing}

Due to its ability to provide $\mathrm{pO}_{2^{-}}, \mathrm{pCO}_{2^{-}}$, lactate-, and potassium values in just a few minutes, POCT using a blood gas analyzer promises to be useful during CA. However, evidence regarding the role of POCT in OHCA is limited. Ahn et al. [50] discovered that POCT was able to detect life-threatening hyperkalemia during CPR with $85 \%$ sensitivity and $97 \%$ specificity. Furthermore, the potassium values measured by POCT were sufficiently similar to values measured in the central laboratory [50]. These results indicate that prehospital POCT could be useful in detecting hyperkalemia during OHCA.

In addition to venous and arterial samples, intraosseous (IO) samples can be used for POCT as well [51-53]. IO samples can be a good alternative as arterial and venous samples can be difficult to obtain in a prehospital setting. Lactate, $\mathrm{pH}$, sodium, and calcium values are similar in arterial, venous, and IO samples [51]. However, IO potassium values should be interpreted with caution, as they are higher than in arterial or venous blood samples $[51,52]$. Also, potassium levels during CPR are on average $2.8-4.4 \mathrm{mmol} / \mathrm{L}$ higher than pre-arrest potassium levels in all types of samples. This is why hyperkalemia may be falsely diagnosed in IO samples but can be reliably ruled out [51].

The remaining available literature predominantly describes a prognostic rather than a diagnostic potential.
Table 4. Summary of diagnostic tools and their applicability

\begin{tabular}{lllll}
\hline & $\begin{array}{l}\text { CC } \\
\text { quality }\end{array}$ & $\begin{array}{l}\text { Diagnostic } \\
\text { value }\end{array}$ & $\begin{array}{l}\text { ROSC } \\
\text { prediction }\end{array}$ & $\begin{array}{l}\text { Long } \\
\text { term }\end{array}$ \\
\hline 4H's and 4T's & - & + & - & - \\
ECG & - & - & + & - \\
Capnography & + & - & + & - \\
Ultrasonography & + & + & + & - \\
Cerebral oximetry & - & - & + & + \\
POCT & - & + & + & + \\
\hline
\end{tabular}

CC Quality, ability to monitor chest compression quality; Diagnostic value, ability to detect underlying causes of CA; ROSC, return of spontaneous circulation; ROSC prediction, ability to predict ROSC; Long term, ability to predict long-term outcome; ECG, electrocardiogram; POCT, point-of-care testing.

Kim et al. [16] found that $\mathrm{pCO}_{2}$ values on blood samples taken within the first 4 min of CPR were independently predictive for ROSC. More specifically, patients with an initial $\mathrm{pCO}_{2}$ value $<75 \mathrm{~mm} \mathrm{Hg}$ were 3.3 times more likely to achieve ROSC than patients with higher $\mathrm{pCO}_{2}$ values [16]. In a study by Shin et al. [54], a relationship between $\mathrm{pH}$ and potassium values and survival to hospital discharge was demonstrated, as well as an association between $\mathrm{pH}$ and neurological outcome. Higher $\mathrm{pH}$ levels were associated with a higher percentage of patients surviving to discharge, while increment of potassium levels beyond $3.5 \mathrm{mEq} / \mathrm{L}$ on the other hand resulted in a decreased survival to discharge ratio. Good neurological outcome could be observed if $\mathrm{pH}$ levels were $\geq 6.8$, with the probability of this outcome increasing with increasing $\mathrm{pH}$ levels [54].

According to the ALS protocol, an OHCA with PEA as initial rhythm, should be managed by determining and treating the underlying cause of the arrest. This poses a challenge in a prehospital setting, which is often characterized by a chaotic environment and limited availability of diagnostic equipment. In this setting, the first line of information comes from clinical examination of the patient and questioning bystanders about the current event and the patient's medical history. This information is then linked to the 4H's and 4T's (Table 3) [12]. However, since clinical and anamnestic information is often limited or absent, other instruments are required to diagnose underlying causes. The available tools and their applicability are summarized in Table 4.

ECG is generally accepted, and has been widely implemented, as an indispensable instrument in CA settings as 
it can discriminate between the various initial rhythms of $\mathrm{CA}$ and is able to monitor CPR progression. In addition, ECG appears to be able to predict ROSC by detecting an increase in heart rate and a decrease in QRS width [29]. Its diagnostic potential however is still unclear since there is only limited and conflicting evidence. Further research is required to determine the diagnostic value of ECG.

As with ECG, capnography has the ability to predict ROSC during ALS by detecting increasing $\mathrm{ETCO}_{2}$ values $[14,27]$. Its diagnostic potential is also rather limited since only one study describes its ability to indicate the cause of CA by detecting high initial $\mathrm{ETCO}_{2}$ values in case of asphyxia [17]. In contrast to ECG, however, capnography can be used for prognosis. Patients with $\mathrm{ETCO}_{2}$ values $>10 \mathrm{~mm} \mathrm{Hg}$ are more likely to achieve ROSC than patients with lower values [14, 17-19]. Furthermore, capnography can be used to monitor quality of chest compressions, while ECG cannot $[8,14]$.

Of all instruments available in a prehospital setting, ultrasonography appears to be the most promising in detecting the underlying cause of a CA. It is the only instrument in this review that is able to detect multiple potential underlying causes [34-37]. Like capnography and ECG, ultrasonography is able to predict ROSC by detecting spontaneous cardiac movement, and like capnography (but not ECG), ultrasonography can indicate the expected prognosis by differentiating pseudo PEA and true PEA $[2,20,21]$. Furthermore, ultrasonography appears to be able to monitor chest compression quality much like capnography [55] because ultrasonography is able to directly visualize the area of compression so that hand positioning can be adjusted, resulting in optimal compression of the left ventricle [55].

Currently, there is no literature available on the diagnostic possibilities of cerebral oximetry. Multiple studies have however investigated its prognostic value. Like capnography and ultrasonography, cerebral oximetry has the potential to determine the short-term prognosis [15, 2224]. Moreover, like ECG, capnography, and ultrasonography, cerebral oximetry is able to predict ROSC as well [22]. However, unlike other instruments, it can also determine the expected long-term prognosis and neurological outcome $[49,56]$. Because of this additional ability, cerebral oximetry could become an important asset for prognostication and can assist physicians in determining whether or not resuscitative efforts should be discontinued.

POCT appears to be a promising instrument as well. Like capnography, cerebral oximetry, and ultrasonography, POCT can be used to determine the expected prog- nosis and predict ROSC [16]. In addition, like cerebral oximetry, POCT allows the prediction of long-term and neurological outcome [54]. An additional advantage of POCT is the ability to rule out and diagnose hyperkalemia, which cannot be done with any other diagnostic tool described in this review $[50,51]$.

A literature search based on a predefined search strategy was performed. There may be a risk of studies not appearing due to missing relevant key terms in the search. Moreover, publication bias is possible as unpublished studies were not searched.

\section{Conclusion}

We propose the following approach to OHCA with PEA as the initial rhythm. First, emergency physicians should look for clinical signs and anamnestic information and link this to the $4 \mathrm{H}$ 's and $4 \mathrm{~T}$ 's. Secondly, a portable handheld ultrasonography device should be used to check for myocardial contractions (thereby discriminating between true PEA and pseudo PEA), and to screen for underlying treatable causes. Ultrasonography allows physicians to detect or exclude a variety of underlying causes in an efficient manner, with minimal CPR interruptions. Future studies should compare the various ultrasonographic protocols in terms of their feasibility and their effect on patient survival.

Evidence concerning other diagnostic tools is limited. However, further research is required since their use appears promising.

\section{Conflict of Interest Statement}

The authors have no conflict of interest to declare.

\section{Funding Sources}

The authors received no specific funding for this review.

\section{Author Contributions}

All authors contributed to the conception and design of the work. Acquisition, analysis, and interpretation of data and drafting of the manuscript was done by Van den Bempt S., with input from and critical revision by Wauters L. and Dewolf Ph. Dewolf Ph. supervised, edited, and finalized the manuscript. All authors gave final approval of the manuscript. 


\section{References}

1 Porzer M, Mrazkova E, Homza M, Janout V. Out-of-hospital cardiac arrest. Biomed Pap Med Fac Univ Palacky Olomouc Czech Repub. 2017 Dec;161(4):348-53.

2 Rabjoh J, Quan T, Boniface K, Pourmand A. Pseudo-pulseless electrical activity in the emergency department, an evidence based approach. Am J Emerg Med. 2020 Feb;38(2): 371-5.

3 Berdowski J, Berg RA, Tijssen JG, Koster RW . Global incidences of out-of-hospital cardiac arrest and survival rates: Systematic review of 67 prospective studies. Resuscitation. 2010 Nov;81(11):1479-87.

4 Bergström M, Schmidbauer S, Herlitz J, Rawshani A, Friberg H. Pulseless electrical activity is associated with improved survival in outof-hospital cardiac arrest with initial nonshockable rhythm. Resuscitation. 2018 Dec; 133:147-52.

5 Sasson C, Rogers MA, Dahl J, Kellermann AL. Predictors of survival from out-of-hospital cardiac arrest: a systematic review and metaanalysis. Circ Cardiovasc Qual Outcomes. 2010 Jan;3(1):63-81.

6 Wissenberg M, Lippert FK, FF, Folke F, Hansen CM, Christensen EF, et al. Association of national initiatives to improve cardiac arrest management with rates of bystander intervention and patient survival after out-of-hospital cardiac arrest. JAMA. 2013;310(13): 1377-84.

7 Parish DC, Goyal H, Dane FC. Mechanism of death: there's more to it than sudden cardiac arrest. J Thorac Dis. 2018 May; 10(5):3081-7.

8 Soar J, Nolan JP, Böttiger BW, Perkins GD, Lott C, Carli P, et al. European Resuscitation Council Guidelines for Resuscitation 2015: section 3. Adult advanced life support. Resuscitation. 2015 Oct;95:100-47.

9 Rabjohns J, Quan T, Boniface K, Pourmand A. Pseudo-pulseless electrical activity in the emergency department, an evidence based approach. Am J Emerg Med. 2020 Feb;38(2): 371-5.

10 Saarinen S, Kämäräinen A, Silfvast T, YliHankala A, Virkkunen I. Pulseless electrical activity and successful out-of-hospital resuscitation: long-term survival and quality of life: an observational cohort study. Scand J Trauma Resusc Emerg Med. 2012 Oct;20(1):74.

11 Väyrynen T, Kuisma M, Määttä T, Boyd J. Who survives from out-of-hospital pulseless electrical activity? Resuscitation. 2008 Feb; 76(2):207-13.

12 Truhlář A, Deakin CD, Soar J, Khalifa GE, Alfonzo A, Bierens JJ, et al. European Resuscitation Council Guidelines for Resuscitation 2015: section 4. Cardiac arrest in special circumstances. Resuscitation. 2015 Oct;95:148201.

13 Volpicelli G. Usefulness of emergency ultrasound in nontraumatic cardiac arrest. Am J Emerg Med. 2011 Feb;29(2):216-23.
14 Poon KM, Lui CT, Tsui KL. Prognostication of out-of-hospital cardiac arrest patients by 3-min end-tidal capnometry level in emergency department. Resuscitation. 2016 May; 102:80-4.

15 Fukuda T, Ohashi N, Nishida M, Gunshin M, Doi K, Matsubara T, et al. Application of cerebral oxygen saturation to prediction of the futility of resuscitation for out-of-hospital cardiopulmonary arrest patients: a singlecenter, prospective, observational study: can cerebral regional oxygen saturation predict the futility of CPR? Am J Emerg Med. 2014 Jul;32(7):747-51.

16 Kim YJ, Lee YJ, Ryoo SM, Sohn CH, Ahn S, Seo DW, et al. Role of blood gas analysis during cardiopulmonary resuscitation in out-ofhospital cardiac arrest patients. Medicine. 2016 Jun;95(25):e3960.

17 Grmec S, Lah K, Tusek-Bunc K. Difference in end-tidal $\mathrm{CO} 2$ between asphyxia cardiac arrest and ventricular fibrillation/pulseless ventricular tachycardia cardiac arrest in the prehospital setting. Crit Care. 2003 Dec;7(6): R139-44.

18 Pearce AK, Davis DP, Minokadeh A, Sell RE. Initial end-tidal carbon dioxide as a prognostic indicator for inpatient PEA arrest. Resuscitation. 2015 Jul;92:77-81.

19 Salen P, O'Connor R, Sierzenski P, Passarello B, Pancu D, Melanson S, et al. Can cardiac sonography and capnography be used independently and in combination to predict resuscitation outcomes? Acad Emerg Med. 2001 Jun;8(6):610-5.

20 Breitkreutz R, Price S, Steiger HV, Seeger FH, Ilper H, Ackermann H, et al. Focused echocardiographic evaluation in life support and peri-resuscitation of emergency patients: a prospective trial. Resuscitation. 2010 Nov; 81(11):1527-33.

21 Tsou PY, Kurbedin J, Chen YS, Chou EH, Lee MG, Lee MC, et al. Accuracy of point-of-care focused echocardiography in predicting outcome of resuscitation in cardiac arrest patients: a systematic review and meta-analysis. Resuscitation. 2017 May;114:92-9.

22 Prosen G, Strnad M, Doniger SJ, Markota A, Stožer A, Borovnik-Lesjak V, et al. Cerebral tissue oximetry levels during prehospital management of cardiac arrest: a prospective observational study. Resuscitation. 2018 Aug; 129:141-5.

23 Sanfilippo F, Serena G, Corredor C, Benedetto U, Maybauer MO, Al-Subaie N, et al. Cerebral oximetry and return of spontaneous circulation after cardiac arrest: a systematic review and meta-analysis. Resuscitation. 2015 Sep;94:67-72.

24 Singer AJ, Ahn A, Inigo-Santiago LA, Thode HC, Henry MC, Parnia S. Cerebral oximetry levels during CPR are associated with return of spontaneous circulation following cardiac arrest: an observational study. Emerg Med J. 2015 May;32(5):353-6.
25 Littmann L, Bustin DJ, Haley MW. A simplified and structured teaching tool for the evaluation and management of pulseless electrical activity. Med Princ Pract. 2014;23(1):1-6.

26 Bergum D, Skjeflo GW, Nordseth T, Mjølstad OC, Haugen BO, Skogvoll E, et al. ECG patterns in early pulseless electrical activity-associations with aetiology and survival of in-hospital cardiac arrest. Resuscitation. 2016 Jul; 104:34-9.

27 Pokorná M, Necas E, Kratochvíl J, Skripský R, Andrlík M, Franek O. A sudden increase in partial pressure end-tidal carbon dioxide $(\mathrm{P}(\mathrm{ET}) \mathrm{CO}(2))$ at the moment of return of spontaneous circulation. J Emerg Med. 2010 Jun;38(5):614-21.

28 Desbiens NA. Simplifying the diagnosis and management of pulseless electrical activity in adults: a qualitative review. Crit Care Med. 2008;36(2):391-6.

29 Skjeflo GW, Nordseth T, Loennechen JP, Bergum D, Skogvoll E. ECG changes during resuscitation of patients with initial pulseless electrical activity are associated with return of spontaneous circulation. Resuscitation. 2018 Jun;127:31-6.

30 Bhende MS, Karasic DG, Karasic RB. Endtidal carbon dioxide changes during cardiopulmonary resuscitation after experimental asphyxial cardiac arrest. Am J Emerg Med. 1996 Jul;14(4):349-50.

31 Berg RA, Henry C, Otto CW, Sanders AB, Kern KB, Hilwig RW, et al. Initial end-tidal $\mathrm{CO} 2$ is markedly elevated during cardiopulmonary resuscitation after asphyxial cardiac arrest. Pediatr Emerg Care. 1996 Aug;12(4): 245-8.

32 Prosen G, Križmarić M, Završnik J, Grmec S. Impact of modified treatment in echocardiographically confirmed pseudo-pulseless electrical activity in out-of-hospital cardiac arrest patients with constant end-tidal carbon dioxide pressure during compression pauses. J Int Med Res;38(4):1458-67.

33 Tsou PY, Kurbedin J, Chen YS, Chou EH, Lee MG, Lee MC, et al. Accuracy of point-of-care focused echocardiography in predicting outcome of resuscitation in cardiac arrest patients: a systematic review and meta-analysis. Resuscitation. 2017 May;114:92-9.

34 Breitkreutz R, Walcher F, Seeger FH. Focused echocardiographic evaluation in resuscitation management: concept of an advanced life support-conformed algorithm. Crit Care Med. 2007 May;35(Suppl):S150-61.

35 Hernandez C, Shuler K, Hannan H, Sonyika C, Likourezos A, Marshall J. C.A.U.S.E.: cardiac arrest ultra-sound exam: a better approach to managing patients in primary nonarrhythmogenic cardiac arrest. Resuscitation. 2008 Feb;76(2):198-206. 
36 Testa A, Cibinel GA, Portale G, Forte P, Giannuzzi R, Pignataro G, et al. The proposal of an integrated ultrasonographic approach into the ALS algorithm for cardiac arrest: the PEA protocol. Eur Rev Med Pharmacol Sci. 2010 Feb [cited 2019 Sep 29];14(2):77-88.

37 Atkinson P, Bowra J, Milne J, Lewis D, Lambert $\mathrm{M}$, Jarman $\mathrm{B}$, et al. International federation for emergency medicine consensus statement: sonography in hypotension and cardiac arrest (SHoC): an international consensus on the use of point of care ultrasound for undifferentiated hypotension and during cardiac arrest. Can J Emerg Med. 2017;19(6):459-70.

38 Chua MT, Chan GW, Kuan WS. Reversible causes in cardiovascular collapse at the emergency department using ultrasonography (REVIVE-US). Ann Acad Med Singap. 2017 Aug [cited 2019 Sep 29];46(8):310-6.

39 McConnell MV, Solomon SD, Rayan ME, Come PC, Goldhaber SZ, Lee RT. Regional right ventricular dysfunction detected by echocardiography in acute pulmonary embolism. Am J Cardiol. 1996 Aug;78(4):469-73.

40 Fields JM, Davis J, Girson L, Au A, Potts J, Morgan CJ, et al. Transthoracic echocardiography for diagnosing pulmonary embolism: a systematic review and meta-analysis. J Am Soc Echocardiogr. 2017 Jul;30(7):714-23.e4.

41 Nazeyrollas P, Metz D, Maillier B, Jennesseaux C, Maes D, Tassan S, et al. [Transthoracic echocardiography and diagnosis of acute pulmonary embolism. Change in the diagnostic valve with respect to thresholds of classification]. Arch Mal Coeur Vaiss. 1997 Apr [cited 2019 Oct 20];90(4):463-9.

42 Miniati M, Monti S, Pratali L, Di Ricco G, Marini C, Formichi B, et al. Value of transthoracic echocardiography in the diagnosis of pulmonary embolism: results of a prospective study in unselected patients. Am J Med. 2001 May;110(7):528-35.

43 Jackson RE, Rudoni RR, Hauser AM, Pascual RG, Hussey ME. Prospective evaluation of two-dimensional transthoracic echocardiography in emergency department patients with suspected pulmonary embolism. Acad Emerg Med. 2000 Sep;7(9):994-8.

44 Torbicki A, Pruszczyk P. The role of echocardiography in suspected and established PE. Semin Vasc Med. 2001 Nov; 1(2):165-74.

45 Hayhurst C, Lebus C, Atkinson PR, Kendall R, Madan R, Talbot J, et al. An evaluation of echo in life support (ELS): is it feasible? What does it add? Emerg Med J. 2011 Feb;28(2): $119-21$.

46 Niendorff DF, Rassias AJ, Palac R, Beach ML, Costa S, Greenberg M. Rapid cardiac ultrasound of inpatients suffering PEA arrest performed by nonexpert sonographers. Resuscitation. 2005 Oct;67(1):81-7.

47 Genbrugge C, Dens J, Meex I, Boer W, Eertmans W, Sabbe M, et al. Regional cerebral oximetry during cardiopulmonary resuscitation: useful or useless? J Emerg Med. 2016 Jan; 50(1):198-207.

48 Nishiyama $\mathrm{K}$, Ito $\mathrm{N}$, Orita $\mathrm{T}$, Hayashida $\mathrm{K}$, Arimoto $\mathrm{H}$, Abe $\mathrm{M}$, et al. Characteristics of regional cerebral oxygen saturation levels in patients with out-of-hospital cardiac arrest with or without return of spontaneous circulation: a prospective observational multicentre study. Resuscitation. 2015 Nov;96:16-22.

49 Ito N, Nishiyama K, Callaway CW, Orita T, Hayashida K, Arimoto H, et al. Noninvasive regional cerebral oxygen saturation for neurological prognostication of patients with out-of-hospital cardiac arrest: a prospective multicenter observational study. Resuscitation. 2014 Jun;85(6):778-84.

50 Ahn S, Kim WY, Sohn CH, Seo DW, Kim W, Lim KS. Potassium values in cardiac arrest patients measured with a point-of-care blood gas analyzer. Resuscitation. 2011 Dec;82(12):e25-6.

51 Jousi M, Skrifvars MB, Nelskylä A, Ristagno G, Schramko A, Nurmi J. Point-of-care laboratory analyses of intraosseous, arterial and central venous samples during experimental cardiopulmonary resuscitation. Resuscitation. 2019 Apr;137:124-32.

52 Jousi M, Saikko S, Nurmi J. Intraosseous blood samples for point-of-care analysis: agreement between intraosseous and arterial analyses. Scand J Trauma Resusc Emerg Med. 2017 Sep;25(1):92.

53 Tallman CI, Darracq M, Young M. Analysis of intraosseous blood samples using an EPOC point of care analyzer during resuscitation. Am J Emerg Med. 2017 Mar;35(3):499-501.

54 Shin J, Lim YS, Kim K, Lee HJ, Lee SJ, Jung E, et al. Initial blood $\mathrm{pH}$ during cardiopulmonary resuscitation in out-of-hospital cardiac arrest patients: a multicenter observational registry-based study. Crit Care. 2017 Dec; 21(1):322.

55 Blanco P, Martínez Buendía C. Point-of-care ultrasound in cardiopulmonary resuscitation: a concise review. J Ultrasound. 2017 Sep; 20(3):193-8.

56 Nishiyama K, Ito $\mathrm{N}$, Orita $\mathrm{T}$, Hayashida $\mathrm{K}$, Arimoto H, Beppu S, et al. Regional cerebral oxygen saturation monitoring for predicting interventional outcomes in patients following out-of-hospital cardiac arrest of presumed cardiac cause: a prospective, observational, multicentre study. Resuscitation. 2015 Nov; 96:135-41. 